\title{
Réflexion sur les modalités d'appréciation du rajeunissement in vitro chez le Sequoia sempervirens
}

\author{
Y. Arnaud1', Y. Fouret ${ }^{1}$, C. Larrieu ${ }^{1}$, H. Tranvan¹, A. Franclet ${ }^{2}$ \\ et E. Miginiac ${ }^{1}$
}

1 Université P.-et-M.-Curie, Laboratoire de Physiologie du Développement des Plantes, T 53-E 5, 4, pl. Jussieu, 75252 Paris Cedex 05, et

2 AFOCEL, Domaine-de-l'Etançon, 77370 Nangis, France

\section{Introduction}

Le rétablissement des propriétés "de jeunesse" chez les fragments d'un arbre âgé est un préalable indispensable à la micropropagation in vitro, puis à l'acclimatation des vitroplants (Franclet, 1981; Franclet et al., 1987). Chez le Sequoia sempervirens, neuf protocoles de rajeunissement in vitro ont été mis en œuvre (Fouret et al., 1985; Fouret, 1987). Pour juger de leur efficacité, il fallait disposer d'un certain nombre de critères. Nous avons donc comparé les comportements de matériels issus d'arbres âgés de $1-50$ et 500 ans (clones $\mathrm{J}-\mathrm{I}$ et $\mathrm{II}$, respectivement). Ceci nous a permis de définir des critères morphologiques, physiologiques et biochimiques de l'état jeune, de l'état adulte et de l'état âgé (Fouret et al., 1985; Fouret et al., 1986; Fouret, 1987). Nous avons ensuite éprouvé la validité de ces critères quant aux protocoles de rajeunissement (Fouret et al., 1985; Fouret, 1987). II est apparu que dans un travail de ce type il faut réfléchir aux points suivants.

\section{Eprouver la fiabilité de chaque critère}

Les résultats fournis sont-ils répétables? Sinon, pourquoi?

Deux hypothèses sont alors à envisager: ou bien le critère n'est pas fiable ou bien il révèle l'intervention de facteurs non encore maîtrisés. Ainsi, nous référant au critère "enracinement induit" (milieu d'induction avec ANA $5 \times 10^{-5} \mathrm{M}$ pendant une semaine, puis milieu d'expression sans hormone pendant 42 jours), nous avons obtenu successivement chez le clone II, au début de trois protocoles de rajeunissement, $67 \%, 25 \%$ et $0 \%$ d'explants enracinés (Fouret, 1987). Ceci nous a amené à prendre en compte l'histoire in vitro du matériel antérieure à «la mise en rajeunissement" (non seulement le temps écoulé depuis l'introduction in vitro, mais aussi les modalités d'entretien). De même, quand le séjour sur milieu d'allongement, au cours de l'entretien d'un matériel rajeuni, se prolonge de 8 à 2?. semaines, le pourcentage 
d'enracinement induit passe de 100 à 33 . Cet exemple qui confirme les précédents souligne le caractère réversible des propriétés du matériel que nous considérons comme des caractéristiques de jeunesse.

Peut-on utiliser valablement chaque critère en toutes circonstances expérimentales?

Nous ne mentionnerons pas les conditions d'environnement pour lesquelles les observations sont encore fragmentaires. Nous n'évoquerons que le type de matériel utilisé (Fouret, 1987). Généralement, au cours d'un protocole de rajeunissement, pour un matériel issu d'un arbre de 500 ans, le pourcentage d'enracinement spontané demeure voisin de zéro. Par contre, le pourcentage d'enracinement induit révèle des changements de l'état physiologique: par exemple, il passe de 0 à 66 ou mieux 90; ce critère est donc mieux adapté que le précédent à l'évaluation du rajeunissement d'un matériel issu d'un arbre âgé. Au contraire, pour un matériel issu d'un arbre de 50 ans, le pourcentage d'enracinement induit demeure voisin du maximum; c'est le pourcentage d'enracinement spontané qui révèle un degré supplémentaire de rajeunissement: il passe de 0 à 17, par exemple. Ceci souligne qu'un critère doit être adapté et qu'il est prudent de ne pas se limiter à un seul.

La validité d'un critère se maintient-elle pendant la durée d'une expérience de rajeunissement? Sinon pourquoi?

Dans un ensemble de trois protocoles (Fouret, 1987), le pourcentage d'enracinement induit révèle un rajeunissement du matériel adulte (clone l) et du matériel âgé (clone II); ceci est confirmé par plusieurs autres critères. Par contre, chez le clone $\mathrm{J}$, le pourcentage d'enracinement induit augmente d'abord, puis chute brutalement au cours de l'expérimentation; il en est de même pour le nombre de racines. Faut-il penser que le matériel, initialement jeune, rajeunit dans un premier temps, puis vieillit brusquement? Les résultats fournis par les critères "délai d'enracinement induit", "hauteur de la tige" et "néoformation spontanée de bourgeons épiphylle" vont à l'encontre de cette hypothèse. Faut-il penser que "pourcentage d'enracinement induit" et "nombre de racines" ne sont pas des critères fiables? Les très nombreuses observations effectuées depuis cinq ans (Bekkaoui et al., 1984; Fouret, 1987) montrent que tel n'est pas le cas. L'intensité de rhizogenèse qui exprime un état de jeunesse physiologique pourrait être limitée par la diminution - voire la disparition - d'un facteur biochimique ou par une non-compétence des tissus. Ainsi, le fait qu'un critère paraisse perdre sa validité au cours d'un protocole de rajeunissement peut révéler l'intervention de facteur(s) qu'il convient de déterminer; ceci souligne aussi l'importance d'utiliser simultanément plusieurs critères.

\section{S'interroger sur les modalités d'expres- sion de certains critères}

Le critère "aptitude à l'enracinement" peut être appliqué à l'enracinement spontané ou à l'enracinement induit; de plus, il peut être exprimé selon quatre modalités: "pourcentage d'explants enracinés", "délai d'apparition de la première racine", "nombre moyen de racines par explant", «type de système racinaire». Peut-on se référer à une seule de ces modalités? Du matériel âgé (clone II) est soumis à trois 
protocoles de rajeunissement pendant 18 semaines (Fouret, 1987). Dans le premier, aucun rajeunissement n'est décelé. Dans le second, le rajeunissement est mis en évidence par les quatre modalités, mais les résultats fournis par la quatrième sont moins nets que les résultats fournis par les trois autres. Dans le troisième protocole, un rajeunissement est décelé seulement par le pourcentage et par le délai d'enracinement induit. Dans ce dernier cas, le rajeunissement est moins accentué que dans le précédent; si l'on ne se référait qu'à une ou deux modalités, on pourrait ne rien déceler et conclure à l'inefficacité du protocole.

\section{S'interroger sur la quantité et la qualité de l'information que fournissent les dif- férents critères}

Au cours d'un protocole de rajeunissement, l'aptitude à l'enracinement induit s'exprime plus tôt que l'aptitude à l'enracinement spontané. Dans certains cas, alors que les pourcentages optima d'enracinement induit et spontané sont atteints, une aptitude au bourgeonnement épiphylle spontané apparaît (Fouret, 1987). II semble que le rajeunissement s'accentue progressivement. Pourtant, on observe parfois, en fin de protocole, une baisse des pourcentages d'enracinement et du nombre de racines. Le rajeunissement est, dans la pratique, considéré comme "total" s'il y a croissance orthotrope de la tige après transfert ex vitro. Des vitroplants, issus du bourgeonnement épiphylle induit de matériel âgé (clone II) ont une croissance orthotrope, en serre, depuis un an. Nous pouvons dire que le rajeunissement "total" a été obtenu, sous réserve que la conformité du matériel soit vérifiée (Franclet et al., 1987). Mais ces vitroplants ontils conservé la «totalité» des propriétés du matériel rajeuni?
Tenir compte des avantages et des inconvénients des différents critères

Se référer au critère "enracinement spontané" présente des avantages sur le plan pratique: on fait l'économie des repiquages sur le milieu d'induction et le milieu d'expression de la rhizogenèse; la lecture des résultats n'exige pas un long délai et l'enracinement est de bonne qualité (racines longues et ramifiées). Mais il ne faut pas oublier un inconvénient majeur: l'aptitude à l'enracinement spontané ne s'exprime que tardivement au cours d'un protocole de rajeunissement et elle est généralement faible. II est donc nécessaire de se référer à d'autres critères pour avoir rapidement des informations suffisantes sur l'efficacité d'un protocole.

\section{Vérifier s'il existe une corrélation entre les différents critères, particulièrement entre des critères physiologiques et des critères bilochimiques}

II est intéressant de mettre en évidence une corrélation entre plusieurs critères: en effet, la validité de chacun s'en trouve confirmée; de plus, un critère peut, éventuellement, être choisi à l'exclusion des autres en raison de sa spécificité: simplicité d'utilisation ("croissance de la tige"), précocité d'expression et rapidité d'évaluation («délai de réactivation des méristèmes caulinaires isolés"), précision et intérêt d'ordre fondamental («rapport AIA $A B B A$ endogènes"), intérêt d'ordre appliqué ("aptitude à l'enracinement»); enfin, une approche des mécanismes du rajeunissement peut être amorcée: par exemple, il est intéressant de chercher à établir une corrélation entre l'évolution de l'aptitude à l'enracinement et l'évolution du rapport AIA ABA (Fouret, 1987). Ainsi, chez le clone II, au cours d'un protocole 
de rajeunissement, le rapport $A \mid A / A B A$ passe de 0,17 à 0,31 en 18 semaines; parallèlement, le pourcentage d'enracinement induit passe de 0 à 66; dans un protocole plus efficace, le rapport AIA $A B A$ s'élève dans le même temps à 4,63; parallèlement, le pourcentage d'enracinement induit atteint 100 .

Quand des critères ne sont pas corrélés (Fouret, 1987), il faut s'interroger sur le type d'information qu'ils peuvent néanmoins fournir.

\section{Exemple 1}

Chez le clone $\mathrm{J}$, au cours d'un protocole de rajeunissement, le rapport $A / A / A B A$, initialement de 0,60 , s'élève à 3,28 en 12 semaines; parallèlement, le pourcentage d'enracinement induit, initialement de 75 , s'abaisse à 42 . Ce résultat paradoxal montre que les propriétés du matériel jeune se modifient rapidement et qu'apparaissent des facteurs limitants.

\section{Exemple 2}

Le délai de réactivation des méristèmes caulinaires isolés révèle parfois des fluctuations apparemment injustifiées de l'état physiologique du matériel (Fouret, 1987). On peut alors se demander s'il s'agit bien là d'un critère de rajeunissement (et de nombreuses expériences permettent de répondre par l'affirmative) ou si ce critère apporte une information complémentaire: par exemple, on sait que chez les arbres, dans les conditions naturelles, il y a apparition de rythmes de croissance au cours du vieillissement; les fluctuations in vitro du délai de réactivation des méristèmes caulinaires isolés ne traduiraient-elles pas la "mise en mémoire" du fonctionnement complexe de l'arbre, acquis au cours du temps?

\section{Conclusion}

Ainsi, le processus de rajeunissement est complexe; il est progressif et plus ou moins rapide selon l'état physiologique initial du matériel traité; en outre, au cours du temps, il y a disharmonie entre les évolutions respectives d'un certain nombre des propriétés qui caractérisent l'âge d'un matériel. L'état rajeuni peut aussi disparaître (du moins certaines de ses propriétés) : il est donc nécessaire de l'entretenir. Alors que nous ne disposons pas encore d'un marqueur incontestable et suffisamment sensible (par exemple protéique) du degré de rajeunissement, nous pensons qu'il est prudent de se référer à plusieurs critères, simultanément, à chaque étape d'un protocole. II convient de rechercher le protocole le plus simple pour lequel le plus grand nombre de critères indique un rajeunissement. Nous avons tenté de définir les plus significatifs en utilisant l'analyse factorielle des correspondances; dans nos conditions expérimentales, ce sont le pourcentage et le délai d'enracinement induit, la hauteur de la tige, le nombre de feuilles, le délai de réactivation des méristèmes caulinaires isolés, le rapport $A I A / A B A$ endogènes. Enfin, la croissance orthotrope de la tige après transfert ex vitro est, dans la pratique, considérée comme le critère du rajeunissement "total". Mais peut-on parler de rajeunissement «total» si certaines propriétés de l'état rajeuni ont pu disparaître au cours même du protocole de rajeunissement? Sans doute, dans la mesure où ces propriétés sont, à chaque étape du processus du rajeunissement, les "marqueurs" d'un état physiologique qui évolue. Toutefois, l'étape ultime du rajeunissement ne devrait-elle pas être «marquée» par l'aptitude à l'embryogenèse somatique? Les recherches en cours et à venir devraient permettre de répondre à ces questions. 


\section{Références}

Bekkaoui F., Arnaud Y., Larrieu C. \& Miginiac E. (1984) Etude comparative de la rhizogenèse in vitro du Sequoia sempervirens chez deux clones d'âge différent. Ann. Rech. Sylv. 1983 AFOCEL, Paris, pp. 5-25

Fouret Y. (1987) Etude in vitro du rajeunissement préalable à la micropropagation chez le Sequoia sempervirens (Endl.) : recherches de marqueurs morphologiques, physiologiques et biochimiques. Thèse de Doctorat de l'Université Pierre-et-Marie-Curie (Paris VI), Paris, France

Fouret Y., Arnaud Y. \& Larrieu C. (1985) Rajeunissement in vitro du Sequoia sempervirens: effet du nombre et de la fréquence des repiquages; recherche de critères précoces de juvénilité. Ann. Rech. Sylv. 1984 AFOCEL, Paris, pp. 111-137

Fouret Y., Arnaud Y., Maldiney R., Sotta B. \& Miginiac E. (1986) Relation entre rhizogenèse et teneurs en auxine et acide abscissique chez trois clones de Sequoia sempervirens (Endl.) issus d'arbres d'âge différent. C.R. Acad. Sci. Paris Sér. II/4, 135-138

Franclet A. (1981) Rajeunissement et propagation végétative des ligneux. Ann. Rech. Sylv. 1980 AFOCEL, Paris, pp. 11-41

Franclet A., Boulay M., Bekkaoui F., Fouret Y., Verschoore-Martouzet B. \& Walker N. (1987) Rejuvenation. In: Cell and Tissue Culture in Forestry. Vol. I: General Application in Biotechnology (Bonga J.M. \& Durzan D.J., eds.), M. Nijhoff and Dr. D.W. Junk (Canada), pp. 232248 